\title{
A Cellular Automata Approach to CFD Flame Spread Modelling
}

\author{
ANTHONY FERNANDO \\ Centre for Environmental Safety and Risk Engineering \\ Victoria University of Technology, Melbourne, Australia
}

\begin{abstract}
This paper focuses on the growth and spread stage of full-scale fires in enclosures, where a localised flame spreads across a single fuel item, increasing the heat release rate, thereby resulting in increased fire hazard. The development of a numerical model for fire spread over a solid fuel surface, and its integration into a CFD model, is described. The conflicting demands of resolving the small scale flame front phenomena while simultaneously accounting for the large-scale enclosure flow phenomena are addressed. The surface of the fuel is descretised with a regular square array, and flame spread occurs as a series of ignitions of surface elements. Ignition of an element is determined by a combination of critical surface ignition temperature and cellular automata techniques. Regression of the combusting fuel surface is modelled, with a fine grid retained at the fuel surface by allowing the grid to "collapse" with the surface. A grid transformation is applied to restore orthogonality of the collapsed grid, for simple computation of the heat equation. The flame spread model has been designed to be independent of geometry, although experimental validation exercises are carried out for radial spread over horizontal surfaces only. Predictions are made for the burning of a slab of polyurethane foam in a full-scale multi-room experimental building-fire facility. The predictions compare favourably with the experiments, indicating the broad validity of the methods used in flame spread model.
\end{abstract}

KEY WORDS: CFD, Field Modelling, Flame Spread, Fire Growth, Cellular Automata.

\section{INTRODUCTION}

Rate of heat release has been identified as an important factor in determining the fire safety hazard in an enclosure of a particular material. The heat release rate in an actual fire is in turn influenced by the conditions the material experiences, including the heat feedback from the surroundings, and the flame spread across the material which increases the area releasing heat. Computational fluid dynamics (CFD) modelling, or fire field modelling, is a useful tool to aid in predicting the possible fire hazard of a particular material. However, despite the continuing advancements in computer processing speed and storage capacity, fire field models still push the capabilities of computers to 
their limits when modelling full-scale unsteady-state enclosure fires, due to the sheer number of calculations required. This is particularly true for a model which is attempting to simulate a large enclosure, in which both small and large scale phenomena are occurring, such as the progression of a flame front under the heating influence of a hot combustion product layer. In the past, modellers have tended to concentrate on one of the other of these phenomena; for example, if the enclosure as a whole is being modelled, then the heat release rate is often prescribed [1], and if flame spread is considered, then it is usually in the form of an empirical equation [2], or restricted to a special case, such as vertical spread up a wall. On the other hand, if flame spread is concentrated upon in detail, it is often in isolation, with conditions prescribed for the fire environment [3].

This paper describes the construction of a flame spread model in such a way that it operates in conjunction with a CFD model to predict full-scale fires involving flame spread across solid fuel objects. The flame spread model contributes heat and fuel volatiles to the gas phase, whereby the CFD model calculates combustion of the fuel vapours, and distributes the heat and products of combustion accordingly. At the same time, the CFD model calculates the heat feedback to the fuel surface within the flame spread model, in turn affecting the flame spread rate and rate of volatilisation of the fuel.

\section{MODEL DESCRIPTION}

\section{Model Requirements}

It has been shown previously [4] that it is possible to produce reasonably accurate results from an isolated flame spread model that makes only basic assumptions about the gas phase phenomena. Inaccuracies in the isolated flame spread model were largely attributed to these assumptions, which are not flexible enough to adequately simulate the changing conditions occurring in a fire growth scenario. Fire field models on the other hand are designed to simulate the gas phase phenomena occurring in a combustion scenario, given a heat or fuel source.

There are several requirements of such a flame spread submodel, which need to be taken into account if is to be generally compatible with a CFD model. For compatibility, the flame spread model will operate on a discrete grid. This means that unlike most analytical models of flame spread, the flame front will be characterised as advancing by a series of discrete jumps rather than continuously. The requirements of the flame spread model are basically threefold. Firstly, like CFD models, the flame spread model should be based as much as possible on first principles. Secondly, it must be geometrically flexible, so that it is capable of modelling a wide variety of flame spread scenarios. Thirdly, the fundamental material properties required for the model must be experimentally derivable [5].

In a recent paper, Nicolette et al. discussed the necessary coupling between combustion and flame spread models, and general CFD fire models [6]. While their analysis appears commonsense, the authors nevertheless provide a useful summary of the problem, and the essence of the analysis may be reduced to four main points. The first point concerns the problem of tracking creeping (ie. opposed flow) flame spread, which requires modelling of small-scale phenomena, in a large enclosure. Since modelling of a large enclosure requires a coarse grid if computational resources are not to become prohibitive, it was proposed that a solution should track the creeping spread by empirical or other means on a subgrid of the CFD grid. The second point concerns the representation of the fuel in the computational region, either as a solid object or a wall lining for 
example, and how to model the changing shape of the fuel as combustion progresses. Thirdly, the coupling of enthalpy and mass source terms was identified as being sensitive in field models. Any imbalance will result in mass and energy no longer being conserved. Hence, accurate coupling is important. The fourth point concerns the choice of grid for the finite difference (or finite element) method used in both the flame spread and field models. A suggestion was for a grid for the solid fuel which is refined at the surface near the flame front, and which moves with the front.

These four issues are dealt with to varying degrees in the method presented herein. The need for a fine grid to model opposed flow flame spread was previously identified by the author [4], and indeed the method chosen here is to construct a grid for the flame spread model which is finer than that used in the CFD model. The flame spread within the larger CFD grid cells is modelled using a cellular automata techniques, explained later. The fuel is modelled as an obstacle within the flow region, with combustion and mass loss occurring at one surface only. Regression of the fuel surface is taken into account in the flame spread model, as a first approximation, the fuel obstacle retains its shape in the CFD model, mainly because the CFD model presented here operates on a fixed orthogonal grid. The author anticipates that while the regression of the fuel surface is important to the heat conduction within the fuel, the effect will be negligible in terms of the largescale behaviour of the flow region. The products of pyrolysis of the solid fuel are not modelled in any great detail. The only property of the gaseous fuel considered here is heat of combustion, which is assumed constant throughout the execution of the model. The thermodynamic properties of methane are assumed to be sufficiently representative of the gaseous fuel. This assumption has been used with some success in past modelling of polyurethane combustion [2,7].

\section{The CFD Flame Spread Model}

The field model CESARE-CFD has been developed in recent years at the Centre for Environmental Safety and Risk Engineering (CESARE) to model a number of fire experiments which have been performed at the Centre's Experimental Building-Fire Facility (EBFF) [7,8]. It is a modified version of the field model FURNACE, originally developed by Boyd and Kent to model coal fired furnaces and pool fires [9]. The model solves the Navier-Stokes and general conservation equations in three dimensions, with the pressure-momentum equations being solved by the SIMPLER algorithm. The standard $k-\varepsilon$ model is used to calculate turbulence, incorporating additional equations to account for turbulence induced by buoyant flows. The quantities solved, and the values for the exchange coefficients and source terms for these quantities are described elsewhere [7].

The combustion of the fuel is modelled by computing the local fuel mixture fraction, then reacting the fuel with the air. The local combustion rate is governed by the local mixing rate of fuel with air. When the local oxygen concentration falls below a specified value (typically in the region of $5-10 \%$ ), combustion is assumed not to occur. A simple empirical equation is used to calculate the composition of combustion products [7]. Radiation heat transfer is calculated using the discrete transfer method [10]. As the radiation calculations are particularly time consuming, the calculations are performed on a coarser grid than used for the flow variables, and performed once for every five iterations of the flow region calculations.

The grid itself, both in the flow subroutine and in the radiation subroutine, is rectilinear, with cell boundaries perpendicular to one of the three Cartesian axes. Since most enclosures in architecture are rectilinear, particularly those associated with domestic and office buildings, the rectilinear grid is quite adequate for such applications. The grid spacing in each of the three directions may be 
specified, so that the grid may be refined in required regions, for instance in regions where high temperature gradients are expected.

\section{The Cellular Automata Flame Spread Technique}

Most flame spread models either calculate the spread rate explicitly [11], or the position of the flame front is determined by interpolating between points which are undergoing combustion, and those which are not [12]. Such models rely on assumptions about the geometry or configuration of the combustion taking place. In most cases, these models share the concept that the arrival of the flame front coincides with the fulfilment a determining criterion, usually the attainment by the surface of a critical temperature. The model presented here differs from these other models in that it does not explicitly calculate the flame front location or velocity. Spread occurs by a series of ignitions of surface elements, or cells, whose temperature has reached a critical temperature. This temperature does depend, though, on the ignition status of neighbouring cells. The term "neighbouring" refers to the eight cells on a square grid which surround the central square.

The model presented here considers two types of ignition, namely autoignition and piloted ignition. Autoignition of a cell will occur when the surface temperature of the cell is raised to a sufficiently high value, independent of the ignition status of neighbouring cells. Piloted ignition of a cell may occur at a temperature significantly lower than that for autoignition. In the case of a spreading flame, the pilot is provided by the flame front. For piloted ignition in this model, it is necessary to decide whether the flame front is in the vicinity of the cell in question. The spreading flame is treated as a cellular automaton, and the condition imposed that for

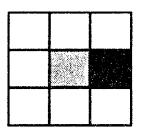

Flame front has not reached cell

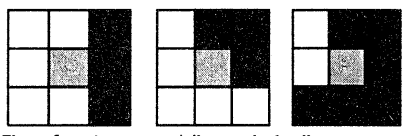

Flame front has (potentially) reached cell

$\square \square$ Unignited cell

Figure 1 Cellular automata criterion for the arrival of the flame front. Figure 1. The three or more neighbour criterion is chosen because, in the absence of other physical criteria, this will lead to a roughly circular spreading flame front [4].

\section{Solid Pyrolysis Model}

The fuel is taken to be a regular rectangular slab. Combustion and mass loss is assumed to occur at one surface only. The other surfaces are assumed to be perfectly insulated in terms of both heat and mass loss. The flame spread is determined by considering the ignition and combustion properties of discrete elements on the fuel surface, with spread occurring as a series of discrete ignitions of fuel elements adjacent to the existing flame front, as described above.

Each element on the combustion surface is in one of three states; preheating, combusting, or burnt out. In the preheating stage, the element is heated remotely by radiation from the flame and external sources. In the burnt out stage, the element is no longer a part of the calculation region, and neighbouring cells are treated as boundary cells. In the combusting stage, the element is assumed to receive at least a minimum heat flux total from the flame and external radiation, for reasons discussed later. The rate of mass loss, $\dot{m}^{\prime \prime}$, is given by the simplified equation for steady combustion [13]

$\dot{m}^{\prime \prime}=\frac{\dot{q}_{\text {flame }}^{\prime \prime}+\dot{q}_{\text {external }}^{\prime \prime}-\dot{q}_{\text {loss }}^{\prime \prime}}{H_{v}}$ 
where $\dot{q}_{\text {flame }}^{\prime \prime}$ is the heat flux from the flame, $\dot{q}_{c: t e r m a l}^{\prime \prime}$ is the externally applied flux, $\dot{q}_{\text {loss }}^{\prime \prime}$ is the radiation and conduction losses, and $H_{v}$ is the latent heat of volatilisation. While steady burning seldom occurs under real fire conditions, this expression for the flame heat is nevertheless useful for describing the instantaneous heat balance at the surface.

The temperature at the surface, $T_{0}$, is calculated by considering the heat flux balance at the surface. This balance takes into account the incident radiant heat flux to the surface, $\dot{q}_{\text {external }}^{\prime \prime}$, convective heat transfer to the surface, $\dot{q}_{c o m v e c t}^{\prime \prime}$, conduction of heat into the fuel, $\dot{q}_{\text {conduct }}^{\prime \prime}$, reradiation from the surface, $\dot{q}_{r r}^{\prime \prime}$, and latent heat of volatilisation of the fuel. The conduction heat flux at the surface is proportional to the temperature gradient at the surface, as given by

$\dot{q}_{\text {crmulut }}^{\prime \prime}=-\left.k \frac{\partial T}{\partial z}\right|_{i=0}$

The gradient is approximated by a parabolic fit of the temperature of the surface node and the first two nodes below the surface. Convective heating is modelled by the expression

$\dot{q}_{\text {conrert }}^{\prime \prime}=h\left(T_{\text {gas }}-T_{0}\right)$

The heat transfer coefficient, $h$, is calculated using the wall function method, as described by Launder and Spalding [14]. The mass flux of volatiles from the surface is assumed to obey the simple Arrhenius equation

$\dot{m}^{\prime \prime}=A \exp \left(\frac{-E}{R T_{0}}\right)$

where $E$ is the activation energy and $A$ the pre-exponential constant for the fuel, and $R$ is the universal gas constant. The re-radiant heat loss from the fuel is given by the expression

$\dot{q}_{r r}^{\prime \prime}=\varepsilon \sigma T_{0}^{4}$

Equations 2-5 are substituted back into Equation 1, to derive an expression for the surface temperature, $T_{0}$, which is solved using the Newton-Raphson method. Once surface temperatures are solved, the interior temperatures are solved by the tri-diagonal matrix algorithm, using the surface temperature as a boundary condition. Regression of the fuel surface is taken into account by means of a grid transformation, details of which appear elsewhere [4].

\section{Grid Embedding}

Incorporation of the flame spread model in the CFD model requires embedding the flame spread grid as a subgrid of the CFD model's main flow grid. There are two issues to be considered here. First, quantities which are transferred from the fuel to the flow region need to be either summed or averaged, depending on the quantity. Secondly, quantities transferred from the flow region to the solid fuel need to be distributed over the solid region.

Consider first transfer from the solid fuel to the flow region. The solid contributes gaseous fuel to the flow region. This manifests itself as mass and enthalpy source terms in the flow cell immediately adjacent to the solid. Since the flow grid will in general be coarser than the solid grid, a flow cell adjacent to the solid surface will be in contact with several solid cells. The source terms in the flow cell will simply be the sum of the contributions from all the solid cells with which it is in contact. The heat flux from the fuel surface to the flow region must be treated differently. The flow cell will receive radiated and conducted heat from the fuel surface, and since each surface cell in contact with the flow cell will, in general, be a different temperature, the net contribution from all the cells is not a straightforward calculation. As a first approximation, the 
fuel surface temperature of is chosen to be that of the solid fuel element which coincides with the centre of the flow grid cell.

The other transfer to be considered is heat flux from the flow region to the solid, which is dependent largely on the properties of the flow cells in contact with the fuel surface. An individual solid surface cell will be in contact with only one flow cell, so the obvious choice is to assume the properties of the flow cell prevail at the surface point of interest. However, preliminary modelling exercises using this approach revealed that an "image" of the flow grid could be seen in the results for the solid submodel, due to similar conditions prevailing over blocks of solid cells. Such a situation is undesirable, as it defeats the purpose of using a subgrid of the flow grid, which is to model phenomena whose mechanisms operate on smaller scale than resolved by the flow grid. If the behaviour of the subgrid is dominated by the large scale grid, then there is no gain in information.

Further information about the flow region may be extracted by interpolating between the known values at the discrete flow cell nodes. A simple linear interpolation method is used here, as it is in agreement with the assumed piecewise linear profile between flow nodes used in the derivation of the CFD equations. The quantity of interest distributed throughout the flow region is denoted $\phi$. The surface cell under consideration is at $(x, y)$, where $x$ and $y$ are coordinates in the two directions parallel to the fuel surface, and is in contact with a particular flow cell. The value of the quantity in the flow cell is denoted $\phi_{\mathrm{i}, \mathrm{j}}$, as calculated for the node located at $\left(x_{\mathrm{i}}, y_{\mathrm{j}}\right)$. In general, each flow cell will have 8 neighbours likewise in contact with the surface, as shown in Figure 2.

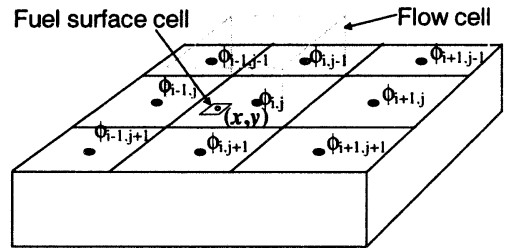

Figure 2 Flow cell neighbours

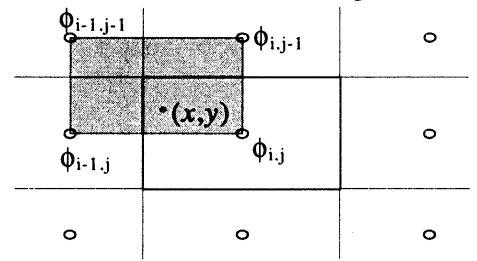

Figure 3 Linear interpolation method

If the point at $(x, y)$ coincides with the flow node, then the value of $\phi(x, y)$ is equal to $\phi_{\mathrm{i}, \mathrm{j}}$. If it is located a finite distance from the node, the value will be somewhere between $\phi_{\mathrm{i}, \mathrm{j}}$ and the value at the nearest neighbours.. The point at $(x, y)$ will in general be located between four flow cell nodes, as shown in Figure 3. The linear interpolation method produces a continuous function with a single equation in two dimensions, and for the example in Figure 3 is given by

$\phi(x, y)=\frac{\left(x_{i}-x\right)\left(y_{j}-y\right) \phi_{i-1, j-1}+\left(x_{i}-x\right)\left(y-y_{j-1}\right) \phi_{i-1, j}+\left(x-x_{i-1}\right)\left(y_{j}-y\right) \phi_{i, j-1}+\left(x-x_{i-1}\right)\left(y_{j}-y\right) \phi_{i, j}}{\left(x_{i}-x_{i-1}\right)\left(y_{j}-y_{j-1}\right)}$

The linear interpolation method is used to determine the incident radiation flux distribution to the fuel surface from the surroundings, since the flux is calculated by the discrete transfer method for the flow nodes only. This method is also used to describe the local values of the flow variables at the fuel surface. These are used to calculate the convective heat transfer to the surface, using the wall-function method. The local heat transfer coefficient for an element on the fuel surface is calculated by first linearly interpolating the flow variables that appear in the wall function equation, and then evaluating the equation. 
Computer modelling of an unsteady-state fire with the CFD model used here is performed in two stages. First, the distribution of variables within the flow region is "initialised" by solving a small steady state fire located at the origin of initiation of the unsteady fire. After convergence of the solution for this small steady-state fire has been attained, the unsteady state simulation is calculated by a forward marching time solution. The flame spread model is by design an unsteady-state model, with the forward marching time solution being calculated from an initial starting point. The starting point is an initially uniform ambient temperature for the solid fuel, a small area of which is ignited. However, incorporation of the flame spread model into the CFD model requires that the fuel be part of the steady-state initialisation process, due to the sensitivity of the coupling between the solid fuel and the flow region. Therefore, conditions need to be specified for the fuel during the steady-state phase of the modelling.

For the steady state conduction of heat in an infinite slab of finite thickness and constant conductivity, the temperature distribution within the slab is a linear temperature profile between the two surfaces. However, it usually takes a long time (dependent on conductivity and thickness) for the temperature within the slab to assume an approximately linear profile. For the flame spread model, the unsteady state will commence with the nearly instantaneous heating of a portion of the upper surface, with the rest of the fuel remaining approximately at ambient temperature; a situation which reflects reality. As a consequence, only surface temperatures are calculated for the flame spread model in the steady state, and the conduction into the fuel is ignored.

Another issue to be addressed is the maintaining of a steady burning region on the fuel surface in the steady-state. The region of the surface designated as ignited must satisfy the criteria for ignition at the conclusion of the steady-state phase of calculations; that is, in the case of this model, the surface must be at least at the critical ignition temperature. If the criteria are not met, the flame spread scenario will not have been properly initiated, and flame spread may not occur. Indeed, combustion may even cease entirely, as insufficient fuel will be contributed to the flow region, resulting in less heat feedback. Maintenance of the ignition criteria is accomplished by prescribing a minimum heat flux to a fuel surface cell that has ignited. This value is the quantity $\dot{q}_{\text {flame }}^{\prime \prime}$ which appears in Equation 1.

Despite the inclusion of a minimum flux to ensure no extinction in the steady-state phase of the calculations, a rapid drop in temperatures throughout the flow region frequently occurred soon after the commencement of the unsteady-state calculations, effectively causing extinction of the fire. This may be due to the somewhat artificial imposition of the minimum heat flux criterion. For this reason, the minimum flux criterion was retained in the flame spread model for the unsteady phase of the calculations.

\section{FOAM SLAB EXPERIMENTS AND MODELLING}

\section{Experiments}

A series of experiments was performed at the EBFF to provide data on the burning of a polyurethane slab in a full-scale enclosure for comparison with the CESARE-CFD flame spread model. The experiments took place in the section of the ground floor shown in Figure 4. 
The layout of the facility comprised a large enclosure connected to the outside via a doorway and corridor, with a window measuring installed at the end of the room opposite the doorway. The floor of the burn room comprised two mass loss platforms, only one of which was required for these experiments.

A series of experiments were performed on standard cushionsized slabs of polyurethane foam, measuring $560 \times 560 \times 100 \mathrm{~mm}$, wrapped in a layer of aluminium

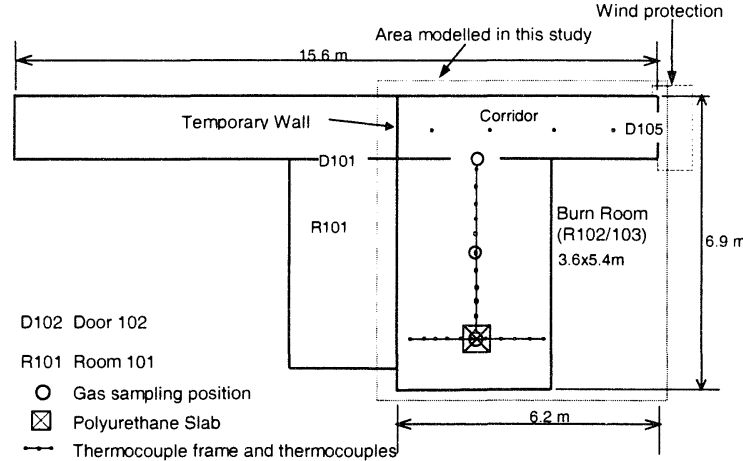

Figure 4 The portion of the Experimental Building-Fire Facility used in this study

foil, and placed horizontally in a holder consisting of five pieces of fire-rated plasterboard (one bottom and four sides, sealed at the joints) assembled so that the foam slab fitted snugly inside, flush with the top of the holder. A grid was marked on the top surface of the foam slab, for visual measurement of flame size from video recordings. Ignition of the foam sample was achieved by the ignition of a small solid fuel tablet placed in the centre of the foam slab. The test was performed three times, with the three tests showing excellent repeatability. An array of thermocouples, gas sampling tubes, velocity probes, and a heat flux meter were used to record a variety of physical quantities at a variety of locations throughout the enclosure in each test.

\section{Modelling}

The portion of the Experimental Building-Fire Facility shown in Figure 4 was the subject of a numerical modelling exercise undertaken with the CFD flame spread model. The enclosure is modelled in the flow region with a $40 \times 54 \times 25$ adaptive grid, which includes a refined region of $14 \times 14$ grid cells adjacent to the fuel slab. As the fuel slab measures $560 \times 560 \mathrm{~mm}$, this corresponds to a $40 \mathrm{~mm}$ grid in the fine region. There are two refined regions in the vertical direction; adjacent to the upper surface of the fuel, and at the midplane of the enclosure, where it is anticipated the interface between the hot and cold interfaces will occur.

The flame spread model discretises the fuel with a $5 \mathrm{~mm}$ grid size, resulting in a $112 \times 112$ grid, and with 8 divisions in the fuel depth, increasing in size from the surface to the depth. The kinetic and thermal properties of the standard polyurethane foam fuel are as given in Table 1. The density and conductivity were measured directly, while the others were taken from typical values in the literature for standard flexible polyurethane foam $[13,15]$, except for the pre-exponential constant, which was determined by trial and error in previous work [4].

The steady-state initialisation of the flow region was performed in 1000 iterations. The initial flame diameter was set to $0.06 \mathrm{~m}$. After

Table 1 Properties of

Polyurethane Foam

\begin{tabular}{|l|l|}
\hline$\rho$ & $23 \mathrm{~kg} / \mathrm{m}^{3}$ \\
\hline$k$ & $0.038 \mathrm{~W} / \mathrm{m} \cdot \mathrm{K}$ \\
\hline$c$ & $1400 \mathrm{~J} / \mathrm{kgK}$ \\
\hline$L_{v}$ & $1.22 \times 10^{6} \mathrm{~J} / \mathrm{kg}$ \\
\hline$\Delta H_{c}$ & $2.27 \times 10^{7} \mathrm{~J} / \mathrm{kg}$ \\
\hline$E$ & $1.25 \times 10^{5} \mathrm{~J} / \mathrm{mol}$ \\
\hline$A$ & $L_{v} \times 1 \times 10^{8}$ \\
\hline$T_{\text {pilot }}$ & $550 \mathrm{~K}$ \\
\hline$T_{\text {auto }}$ & $715 \mathrm{~K}$ \\
\hline
\end{tabular}
initialisation, the unsteady-state fire was modelled for 360 seconds simulation time, using a time step of $0.1 \mathrm{~s}$, which results in a total of 3600 iterations. This was to ensure that the model included the burnout time, which occurred at around 300 seconds in the EBFF tests. 


\section{RESULTS}

The results are shown for the flame spread across the solid fuel item. The effective diameter of the flame is shown in Figure 5. The effective diameter is calculated for a circle whose area is the same as the total area of the ignited cells, in the case of the flame spread model. In the experiment, the diameter was measured directly until the spreading circular front reached the edges of the square fuel slab (diameter $0.56 \mathrm{~m}$ ), after which the diameter was estimated, up to the equivalent diameter of $0.63 \mathrm{~m}$ for a $0.56 \times 0.56 \mathrm{~m}$ square.

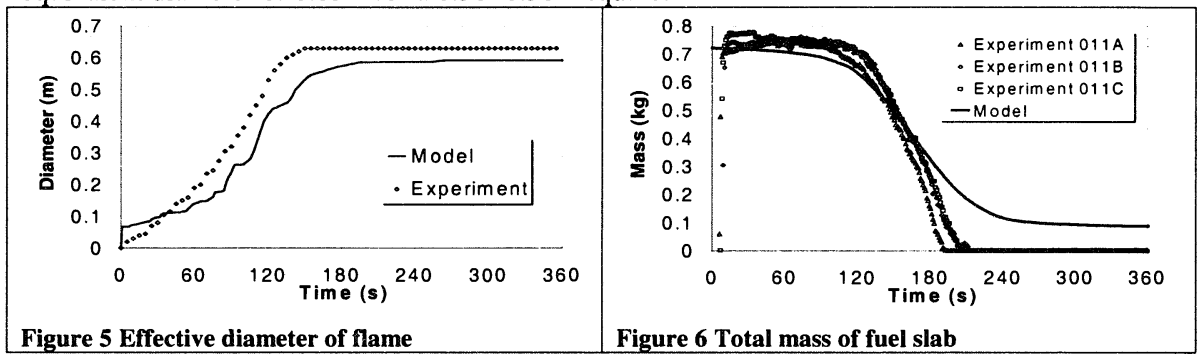

As can be seem from Figure 5, the predicted effective flame diameter failed to reach maximum possible diameter, nor was it predicted that the mass was totally consumed, as shown in Figure 6. Nevertheless, there is reasonable agreement in the overall trends, which suggests that the model is itself reasonable. The model shows the accelerating flame spread, and the timing of the peak mass loss rate corresponds well with the experimentally observed peak in the initial stages of the fire, as shown in Figure 7(a). It is the peak phase and the decay phase of the fire where the modelling results are in disagreement with experimental results. The model fails to predict the sharp peak at 180 seconds, instead predicting a lower, earlier peak with a more gradual decay.
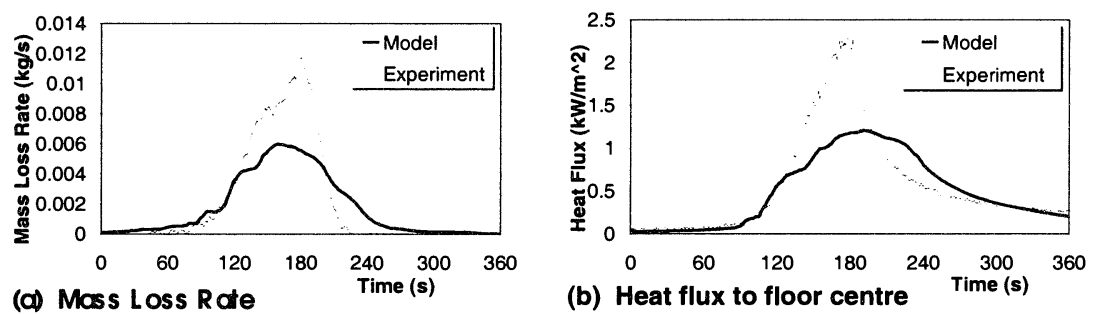

Figure 7 Mass loss and radiation heat flux histories

To understand where the discrepancies are occurring, it is helpful to examine the predictions of the spreading flame in greater detail. The time of ignition of each cell was recorded, and is shown in Figure 8. The light region at the top of Figure 8 corresponds to cells which have not undergone ignition. The initial burning region is the circular region at the centre of the fuel. The flame then spreads radially in a relatively uniform fashion up until around 90 seconds, after which it spreads more rapidly to the south (downwards in the figure) and east, while making little progress northwards, leaving a large portion of the northern edge of the fuel unignited. The flame had spread across the entire surface by $150 \mathrm{~s}$. The model also predicted burnout in the centre at around $120 \mathrm{~s}$, whereas the experiments, with no apparent sign of central burnout, although burnout was difficult to confirm visually. Figure 7(a) shows that this is the point where the predicted mass loss rate becomes noticeably less than the measured mass loss rate. It appears that the discrepancies in 
the mass loss rate and flame diameter curves can be largely attributed to the failure of the model to predict flame spread to the northern portion of the fuel between 120 and 150 seconds.

The unburnt region of the fuel in the simulation is defined by an almost straight line across the northern (top) portion of the fuel. This is the edge of the fuel which faces the doorway of the burn room. The straight line coincides with one of the cell boundaries of the CFD grid. In fact, close examination of Figure 8 reveals evidence of several such straight lines, a phenomenon which was mentioned earlier and led to the inclusion of linear interpolation of flow variables in the flame spread model. The results here show that despite linear interpolation, there is still an "imprint" of the CFD grid on the flame spread grid, of which the northern portion of the fuel is the most dramatic example.

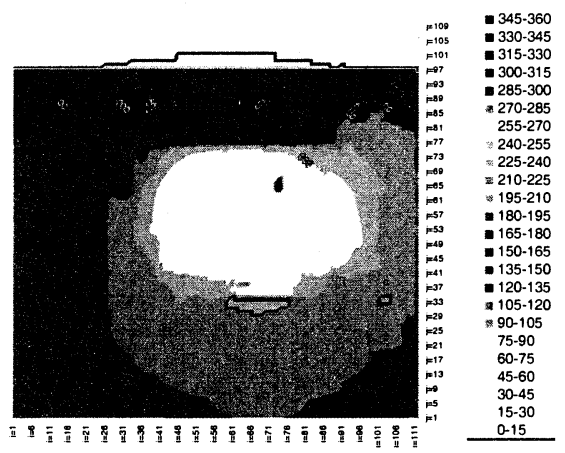

Figure 8 Time of ignition of cells

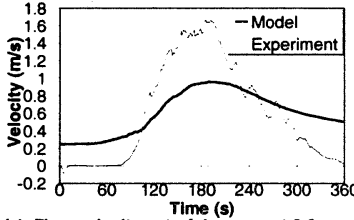

(a) Flow velocity out of doorway at $2.0 \mathrm{~m}$

Figure 9 Velocity histories

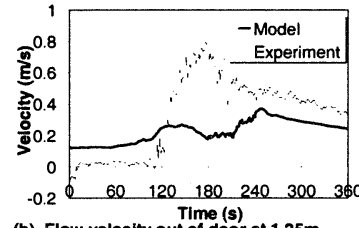

(b) Flow velocity out of door at $1.25 \mathrm{~m}$

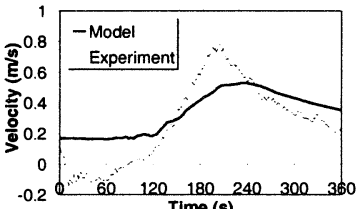

(c) Flow velocity into doorway at $0.25 \mathrm{~m}$

What appears to be causing this predicted phenomenon is the establishment of a strong airflow pattern as combustion proceeds, characterised by the flow of cool air through the lower half of the doorway, across the floor of the burnroom, up the lip of the fuel and across the surface of the fuel, and into the rising plume. This current appears to be strong enough to oppose the spread of flame, or at least to transfer sufficient heat away from the surface to keep the surface temperature below even piloted ignition temperature. In the experiment is, the flame reached each of the four edges within the 120-125 second time interval, indicating that any entrainment and flow pattern created by the fire plume did not show any strong preferential direction up to this time. Examination of the velocity histories in Figure 9 reveals additional information. In the experiment, flows in and out of the doorway were negligible in the first 90 seconds of the experiments and then began to increase noticeably, particularly after 120 seconds. In contrast, there is a significant flow in the simulation from the initial stages, which helps explain the bias to the south in the flame spread simulation. This flow pattern is created by the steady state initialisation of the simulation, and is an issue that will be addressed in future model revisions.

A selection of temperature histories are shown in Figure 10. As with the flow velocities, the steady state initialisation created elevated temperatures at the beginning of the simulation. Overall, the temperatures were generally overpredicted, as is most apparent in Figure 10(c), which shows good agreement in the timing of the temperature peak, but consistently overestimates the 
temperature itself. The temperature histories shown Figure 10(a) and Figure 10(b) again show the delayed and lower peak exhibited by the mass loss rate.

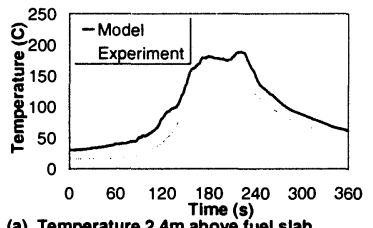

(a) Temperature $2.4 \mathrm{~m}$ above fuel slab

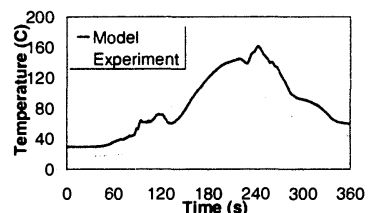

(b) Temperature $1.9 \mathrm{~m}$ above fuel slab

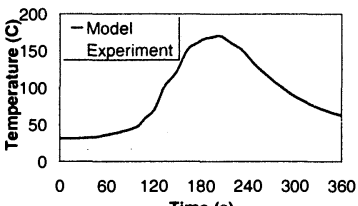

(c) Temperature in doorway at $2.0 \mathrm{~m}$

Figure 10 Temperature histories

Other data recorded were species concentration in the doorway, and in addition oxygen concentration within the enclosure. A sample of points is shown in Figure 11. Oxygen concentration shows excellent correlation between model and experiment in the centre of the enclosure, while above the fuel surface it shows quite a deal of fluctuation, most likely due to the movement of the plume throughout the simulation. Carbon dioxide concentration in the doorway likewise shows good correlation. Overall, there is a tendency for the model to over-predict both $\mathrm{CO}_{2}$ concentration and $\mathrm{O}_{2}$ depletion. However, since the mass loss rate is underpredicted, it is expected that $\mathrm{CO}_{2}$ concentrations should be likewise underpredicted as its source is the mass loss of the fuel. The "extra" carbon is probably an artefact of the steady-state initialisation.

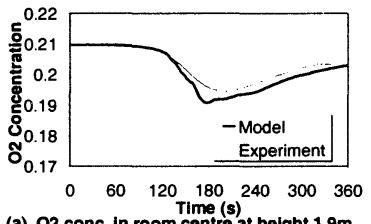

(a) 02 conc. in room centre at height $1.9 \mathrm{~m}$

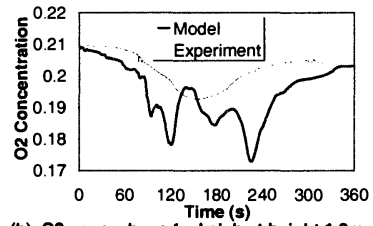

(b) 02 conc. above fuel slab at height $1.9 \mathrm{~m}$

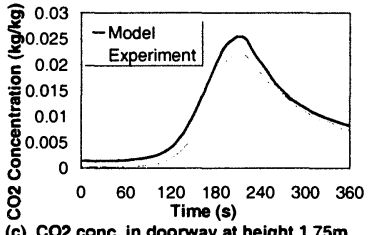

Figure 11 Species concentration at selected locations

The other quantity measured in these experiments was the radiant heat flux to the middle of the burn room floor, as shown in Figure 7(b). The heat flux is underpredicted as would be expected from the mass loss rate. Indeed, the curve for mass loss rate and radiation to the floor show very similar profiles, both for experiment and model, so much so that they appear to be directly proportional. To test this, a simple ratio of heat flux to mass loss rate is calculated and plotted, as shown in Figure 12. This figure shows that for the peak combustion period

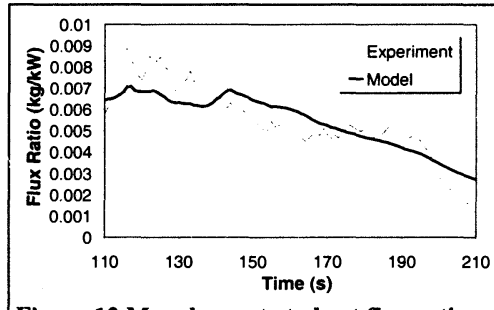

Figure 12 Mass loss rate to heat flux ratio between $120 \mathrm{~s}$ and $210 \mathrm{~s}$, the ratio is quite steady at around $0.006 \mathrm{~kg} / \mathrm{kW}$, and that there is good agreement between model and experiment. In other words, the relatively poor prediction of radiative heat flux is a consequence of the poor prediction of mass loss rate, and hence rate of heat release of the fuel. If the mass loss rate were to have been predicted accurately, the ratio correlation suggests that the radiative heat transfer would likewise have been accurately predicted. 


\section{CONCLUSIONS}

A flame spread model has been incorporated into a fire field model, and has been used to simulate an enclosure fire involving the combustion of a cushion sized slab of standard polyurethane foam. Discrepancies between experiment and modelling have been noted, and an explanation proposed for several of the discrepancies. However, the results overall show reasonably good agreement, which is a favourable outcome for the methods adopted for flame spread modelling presented in this paper. In particular, the cellular automata method is successful in predicting the radial spread of flame from a central ignition point, although the findings presented here show that it is sensitive to the physical data provided by the CFD model. Further refinement of the spread model is required, and further experimental work is desirable, particularly for other fuel configurations. These exercises are also left as future work.

\section{REFERENCES}

1 Hadjisophocleous, G. V. and Cacambouras, M. "Computer Modelling of Compartment Fires", Journal of Fire Protection Engineering, 5 (2), 39-52, 1993

2 Luo, M. and Beck, V. R. "Effects of Air-Handling System on Fire Spread in a Full-Scale Building", Sixth Australasian Heat and Mass Transfer Conference, 241-248, 1996

3 Di Blasi, C., Continello, G., Crescitelli, S. and Russo, G. "Numerical Simulation of Opposed Flow Flame Spread over a Thermally Thick Solid Fuel", Combustion Science and Technology, 54, 25-36, 1987

4 Fernando, A. E. and Thorpe, G. R., "A Numerical Model for Horizontal Flame Spread over Combustible Solid Fuels", Sixth Australasian Heat and Mass Transfer Conference, 213-220, 1996

5 Quintiere, J. and Iqbal, N. "An Approximate Integral Model for the Burning Rate of a Thermoplastic-like Material", Fire and Materials, 18, 89-98, 1994

6 Nicolette, V. F., Tieszen, S. R., Moya, J. L. "Interfacing Materials Models with Fire Field Models", 41st International SAMPE Symposium, March 24-28, 1996

7 Luo, M. and Beck, V. R., "A Study of Non-Flashover and Flashover Fires in a Full-Scale MultiRoom Building", Fire Safety Journal, 1996.

8 Luo, M. and Beck, V. R., "The Fire Environment in a Multi-Room Building-Comparison of Predicted and Experimental Results", Fire Safety Journal, 23, 413-438, 1994

9 Boyd, R. K. and Kent, J. H. "Three Dimensional Furnace Computer Modelling", Twenty-first Symposium (International) on Combustion, 265-274, 1986

10 Lockwood, F. C. and Shah, N. G. "A New Radiation Method For Incorporation in General Combustion Prediction Procedures", Eighteenth Symposium (International) on Combustion, 14051414,1981

11 Baroudi, D., and Kokkala, M., "Flame Spread and Heat Release Rate Model for a Burning Mattress”, Interflam '96, 37-46, 1996

12 Delichatsios, M. M., Mathews, M. K., and Delichatsios, M. A., "An Upward Fire Spread and Growth Simulation", Fire Safety Science: Proceedings of the Third International Symposium, 207216, 1991

13 Drysdale, D. D., An Introduction to Fire Dynamics, John Wiley and Sons Ltd., 1985

14 Launder, B. E. and Spalding, D. B. "The Numerical Computation of Turbulent Flows", Computer Methods in Applied Mechanics and Engineering, 3, 269-289, 1974

15 Chang, T. C., Shen, W. S., Chiu, Y. S., and Ho, S. Y. "Thermo-oxidative Degradation of Phosphorus-containing Polyurethane", Polymer Degradation and Stability, 49, 353-360, 1995 